\title{
Mechanism of Macrophage Action in Osteoarthritis
}

\author{
Yangle $\mathrm{Li}^{1}$, Jie Zheng ${ }^{*}$, Xiaoqing $\mathrm{Wu}^{2}$, Feng Yang ${ }^{3}$ \\ ${ }^{1}$ Shaanxi University of Chinese Medicine, Xianyang 712046, Shaanxi Province, China \\ ${ }^{2}$ Hubei University of Chinese Medicine, Wuhan 430065, Hubei Province, China \\ ${ }^{3}$ Shaanxi University of Chinese Medicine, Xianyang 712046, Shaanxi Province, China \\ *Corresponding author: Jie Zheng, 1483067947@qq.com
}

\begin{abstract}
Osteoarthritis (OA) is the most common chronic joint disease and the main cause of pain and disability in adults (typical clinical OA characteristics), and women are more predisposed to this disease than men. About 400 million people worldwide and more than 100 million in China suffer from arthritis. OA was named the 11th largest contributor of mortality in the world, with a disability rate of as high as 53\%, and is among the three major killers threatening the health of the elderly. Colloquially, OA is called the "number one disabling disease of the 21 st century." It is the main reason for the malfunctioned mobility of the elderly. Generally, women and men start to have OA at 40 and 50, respectively. Incidence rates increased dramatically between the ages of 55 and 60 . The prevalence rate among older persons over 70 years of age is almost $80-90 \%$. In addition, the disease is a chronic progressive disease, which can not only lead to the decline of life function and the reduction or even loss of quality of life, but also has an important and huge impact on health care and social costs. This disease may also demand higher economic requirements of the affected families. Until now, since the pain mechanism of the disease is not clear, there are no effective treatment methods, and surgical joint replacement is the only choice to treat the end-stage disease. This paper focuses on the role of macrophages in OA development, with particular attention to the occurrence of pain and possible mediators involved.
\end{abstract}

Keywords: Osteoarthritis; Macrophage

Publication date: July 2021; Online publication: July 30, 2021

Many studies have confirmed the existence of inflammatory reaction in the synovium of patients with osteoarthritis (OA), and the severity of synovitis is closely related to the severity of OA and the progression of the disease. Synovitis refers to the inflammatory reaction occurring in the synovium of the joint. Patients with OA suffer from pain, and possible mediators are involved in the development of OA ${ }^{[1-3]}$. It is due to occurrence of inflammatory changes. The recognized pathological changes are the proliferation of synovial cells and blood vessels and the infiltration of inflammatory cells ${ }^{[4-6]}$. Macrophages are the main inflammatory cells in synovial tissue, and the proinflammatory factors and cartilage matrix degradation enzymes are found to be important factors that aggravate OA and accelerate joint degeneration ${ }^{[7-9]}$. Many studies have confirmed the existence of macrophages with different activation states in synovial tissues of patients with OA, and these macrophages play an important role in the development of OA ${ }^{[10-18]}$. Therefore, we need to understand the role of synovial macrophages in the occurrence and development of OA, which is particularly important for the understanding of OA and its future treatment.

Macrophages play an important role in osteoarthritis through phagocytosis and acquiring plasticity in phenotype and function, which refers to the transformation from one phenotype to another after stimulation by different molecular patterns. The polarization of macrophages mainly includes two classical activation pathways, M1 (classical activated macrophages) and M2 (selective activated macrophages), which play 
different roles in human body. M1 type induces proinflammatory effect, and Toll-like receptors (TLRs) and interferon gamma (IFN- $\gamma$ ) are stimulated to express inducible nitric oxide synthase (iNOS), CD86 and other genes. Upregulation of inflammatory pathway factors, such as interleukin (IL)-1, IL-6, IL-12, tumor necrosis factor alpha (TNF- $\alpha$ ) and cyclooxygenase 2 (COX-2), participate in and aggravate inflammatory response as well as accelerate cell clearance. M2 type is mainly anti-inflammatory through IL-4- and/or IL13-induced release of IL-10 and transforming growth factor beta (TGF- $\beta$ ) that are involved in inflammation regression, and is conducive to wound healing, and promotes tissue remodeling. M2 can be divided into two types according to its function. M2a can promote cell proliferation and migration; M2b promote cell maturation; M2c accelerate inflammatory regression; M2d can promote angiogenesis [19-21]. The polarization mechanisms of M1 and M2 types are currently unclear. It is recognized that the main known factors are non-associated tyrosine protein kinase/signaling and transcription activator (JAK/STAT), interferon regulatory factor (IRF), notch and phosphatidylinositol-3-kinase (PI3K)/protein kinase B (Akt), etc. ${ }^{[22]}$ More importantly, the polarization conversion of macrophages requires a variety of factors and multiple pathway interactions to achieve, and it is an extremely complex process. The known polarization pathways include JAK/STAT signaling, PI3K/AKT signaling, JNK signaling, MAPK signaling and NF- $\kappa \mathrm{B}$ signaling. The JAK/STAT pathway uses four JAK (another kinase or Janus kinase), namely JAK1, JAK2, JAK3 and Tyk2, plus 7 transcription factor STAT, namely STAT1-4, STAT5A, STAT5B and STAT6; RFM1 and RF-M2 activation of receptors in JAK/STAT pathways. Firstly, JAK/STAT pathway mainly polarizes macrophage M1/M2 through SOCS1 and SOCS3 proteins. Among them, IL-4 activates SOCS1 synthesis to prevent STAT1 blocking, thereby preventing the formation of M1 phenotypes. IFN- $\gamma$ and TLR4 ligand activate SOCS3 synthesis, block STAT3, thus preventing the formation of M2 phenotypes. Meanwhile, STAT6, M2 phenotypic transcription factors SOCS1 activates SOCS3-activated M1 phenotypic transcription factor 1 STAT. Second, JNK pathway is activated by growth factor receptor, cytokine receptor and $\mathrm{G}$ protein-related receptor, which can not only increase the expression of proinflammatory genes, but also activate the SMAD3 of M2 phenotypic transcription factor. There are several main manifestations: (i) controlling macrophage responses to growth factors, cytokines, fatty acids, and G protein-related receptor ligands; (ii) participating in the expression of macrophages into M1 phenotypes; and (iii) activating M2 phenotypic transcription factors SMAD3, thereby limiting the formation of M1 phenotypes. PI3K/Akt-dependent pathway expresses M1 phenotype through Akt2 and M2 phenotype Akt1 expression. PI3K is activated by cytokine receptors and TLR to produce phosphatidylinositol-3,4,5triphosphate (PIP3) and activate protein kinase. Akt1 promotes the formation of M2 phenotypes, while Akt 2 promotes the formation of M1 phenotypes mainly by increasing the expression of iNOS and TNF- $\alpha$ genes of the phenotype. The free NF- $\kappa \mathrm{B}$ is transported to the nucleus to activate the genes involved in inflammation, immune response and cell growth. In addition, I $\kappa \mathrm{B}$ genes can be activated to limit the entry of excessive NF- $\mathrm{B}$ into the nucleus, so as to achieve the purpose of excessive inflammation. Most of the time, TLR/NF- $\kappa \mathrm{B}$ signaling pathways are activated in macrophages M1 phenotypes, increasing proinflammatory cytokine production and reducing anti-inflammatory cytokine production to resist microbial invasion ${ }^{[23]}$.

Macrophage mobile inhibitory factor (MIF) is produced by activated T lymphocytes, which can inhibit macrophage migration and play an important role in human inflammation and immune response system. MIF has been found to play an important role in the repair of chondrocytes. The study found that the MIF and IL-1 $\beta$ in synovial fluid of knee osteoarthritis (KOA) patients were positively correlated, and the expression of IL- $1 \beta$ increased with the severity of the KOA. Therefore, MIF may be an important factor in increasing inflammation of KOA, promoting cartilage degeneration and reducing synovial fluid in joints ${ }^{[24]}$. As we all know, the macrophage activation function of macrophage suppressor (MIF) mainly includes the role of macrophage adhesion, diffusion migration, phagocytosis and promoting the killing of cancer 
cells. Among them, monocytes and macrophages are important sources of enrichment and play an important role in regulating OA. Macrophage inhibitors play a proinflammatory role by stimulating a range of cytokine expression, nitric oxide release, and expression of fibroblast matrix metalloproteinases. In addition, it can antagonize the anti-inflammatory and immunosuppressive functions of pituitary glucocorticoids and regulate the degree of inflammation. It can inhibit the random migration of macrophages and monocytes, and make them aggregate, proliferate and secrete some cytokines at the inflammatory site. Aggregation of macrophages is partially achieved by chemokine (C-C motif) ligand 2 (CCL2) release and induction of other inflammatory factors, such as adhesion molecules and tumor necrosis factor production. MIF interact with inflammatory factors, Inflammatory mediators, such as NF- $\kappa \mathrm{B}$ and $\mathrm{TNF}-\alpha$, promote increased expression. MIF, in turn, can secrete inflammatory cytokines, such as TNF- $\alpha$, IL-6, and IL-1 $\beta$, that are involved in the regulatory process of the inflammatory response ${ }^{[25]}$.

\section{Funding}

Supported by National Natural Science Foundation of China Youth Science Foundation, Shaanxi Provincial Education Department Youth Innovation Team Project (batch number with issue), Shaanxi Provincial Education Department of traditional Chinese medicine prevention and treatment of bone and joint disease research and innovation team.

\section{Disclosure statement}

The author declares no conflict of interest.

\section{References}

[1] Belluzzi E, Stocco E, Pozzuoli A, et al., 2019, Contribution of Infrapatellar Fat Pad and Synovial Membrane to Knee Osteoarthritis Pain. Biomed Res Int, 2019: 6390182.

[2] Vos T, Flaxman AD, Naghavi M, et al., 2012, Years Lived with Disability (YLDs) for 1160 Sequelae of 289 Diseases and Injuries 1990-2010: A Systematic Analysis for the Global Burden of Disease Study 2010. Lancet, 380(9859): 2163-2196.

[3] La Porta C, Bura SA, Negrete R, et al., 2014, Involvement of the Endocannabinoid System in Osteoarthritis Pain. Eur J Neurosci, 39(3): 485-500.

[4] Henrotin Y, Lambert C, Richette P, 2014, Importance of Synovitis in Osteoarthritis: Evidence for the Use of Glycosaminoglycans Against Synovial Inflammation. Semin Arthritis Rheum, 43(5): 579-587.

[5] Liu-Bryan R, 2013, Synovium and the Innate Inflammatory Network in Osteoarthritis Progression. Curr Rheumatol Rep, 15(5): 323.

[6] Scanzello CR, Goldring SR, 2012, The Role of Synovitis in Osteoarthritis Pathogenesis. Bone, 51(2): 249-257.

[7] Bondeson J, Blom Ab, Wainwright S, et al., 2010, The Role of Synovial Macrophages and Macrophage-Produced Mediators in Driving Inflammatory and Destructive Responses in Osteoarthritis. Arthritis Rheum. 62(3): 647-657.

[8] De Lange-Brokaar BJE, Ioan-Facsinay A, Van Osch GJVM, et al., 2012, Synovial Inflammation, Immune Cells and Their Cytokines in Osteoarthritis: A Review. Osteoarthritis Cartilage, 20(12): 1484-1499.

[9] Dai L, Xu C, Tang M, et al., 2003, Effects of Synovial Macrophages on Joint Destruction in 
Adjuvant Arthritis Rats. Chinese Journal of Pathophysiology, 7: 87-90 155.

[10] Cao J, Chen D, 2020, Effects and Characteristics of Synovial Macrophages on Osteoarthritis. China Organization Engineering Research, 29: 4731-4736.

[11] Kraus VB, Mcdaniel G, Huebner JL, et al., 2016, Direct In Vivo Evidence of Activated Macrophages in Human Osteoarthritis. Osteoarthritis Cartilage, 24(9): 1613-1621.

[12] Piscaer TM, Muller C, Mindt TL, et al., 2011, Imaging of Activated Macrophages in Experimental Osteoarthritis Using Folate-Targeted Animal Single-Photon-Emission Computed Tomography/Computed Tomography. Arthritis Rheum, 63(7): 1898-1907.

[13] De Visser HM, Korthagen NM, Muller C, et al., 2018, Imaging of Folate Receptor Expressing Macrophages in the Rat Groove Model of Osteoarthritis: Using a New DOTA-Folate Conjugate. Cartilage, 9(2): 183-191.

[14] Daghestani HN, Pieper CF, Kraus VB. 2015, Soluble Macrophage Biomarkers Indicate Inflammatory Phenotypes in Patients with Knee Osteoarthritis. Arthritis Rheum, 67(4): 956-965.

[15] Wu CL, McNeill J, Goon K, et al., 2017, Conditional Macrophage Depletion Increases Inflammation and Does Not Inhibit the Development of Osteoarthritis in Obese Macrophage FasInduced Apoptosis-Transgenic Mice. Arthritis Rheum, 69(9): 1772-1783.

[16] Zhang H, Lin C, Zeng C, et al., 2018, Synovial Macrophage M1 Polarisation Exacerbates Experimental Osteoarthritis Partially Through R-spondin-2. Ann Rheum Dis, 77(10): 1524-1534.

[17] Athanasou NA, Quinn J, 1991, Immunocytochemical Analysis of Human Synovial Lining Cells: Phenotypic Relation to Other Marrow Derived Cells. Ann Rheum Dis, 50(5): 311-315.

[18] Bloom O, Herman PE, Spungen AM, 2020, Systemic Inflammation in Traumatic Spinal Cord Injury. Exp Neurol, 325: 113143.

[19] Zhou Q, Sun H, Yu D, et al., 2020, Mechanism of M1/M2 Polarization of Macrophages in Different Diseases. China Pharmacology Bulletin, 11: 1502-1506.

[20] Shapouri-Moghaddam A, Mohammadian S, Vazini H, et al., 2018, Macrophage Plasticity, Polarization, and Function in Health and Disease. J Cell Physiol, 233(9): 6425-6440. https://doi.org/10.1002/jcp.26429

[21] Schulert GS, Fall N, Harley JB, et al., 2016, Monocyte MicroRNA Expression in Active Systemic Juvenile Idiopathic Arthritis Implicates MicrorRNA-125a-5p in Polarized Monocyte Phenotypes. Arthritis Rheumatol, 68(9): 2300-2313. https://doi.org/10.1002/art.39694

[22] Xu Y, Xue S, Sang W, et al., 2020, Effects of Macrophage Polarization on Osteoarthritis. Chinese Journal of Bone and Joint, 9: 689-694.

[23] Malyshev I, Malyshev Y, 2015, Current Concept and Update of the Macrophage Plasticity Concept: Intracellular Mechanisms of Reprogramming and M3 Macrophage "Switch" Phenotype. BioMed Research International, 2015: 341308.

[24] Col-Araz N, Oguzkan-Balci S, Baspinar O, et al., 2012, Mannose Binding Lectin and Macrophage Migration Inhibitory Factor Gene Polymorphisms in Turkish Children with Cardiomyopathy: No Association with MBL2 Codon 54 A/B Genotype, but an Association Between MIF-173 CC Genotype. Int J Med Sci, 9(6): 506-12.

[25] Deb, 2009, Mechanism of Macrophage Migration Inhibitory Factor on Cell Proliferation, Migration and Adhesion. Southern Medical University. 